and non-wear times was set at $25^{\circ} \mathrm{C}$. Timeframes during which the temperature rose above $25^{\circ} \mathrm{C}$ were classified as wear time. A Wilcoxon signed-rank test was performed between the different wear times and Spearman's correlations between pain, comfort and wear time were determined.

Results: On average, patients wore the device for $143 \pm 80 \mathrm{~h}$ according to the form they filled out, whereas the TS measured only $83 \pm 86 \mathrm{~h}$, leading to an overestimation of $72 \%$. Patients reported an average pain level of $3 \pm 1.4$ during the six weeks period and the AFO's comfort was rated with $1.9 \pm 0.3$.

Statistical differences were found between the wear time reported by the patients and the wear time derived from the TS $(p=0.005)$. Additionally, a significant correlation between the AFO comfort and the wear time derived from the TS was found $(r=-0.81 ; p=0.001)$. No significant correlation was found between pain and wear time.

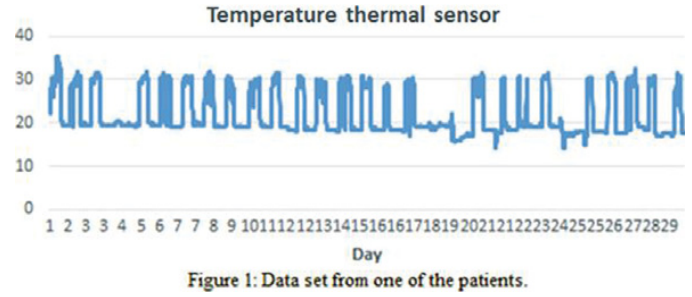

Conclusions: As can be expected, patients who found the AFO more comfortable were the ones who wore the orthosis for longer periods. The wear time recorded by the TS was significantly lower compared to the self-reports. This might be due to a social desire bias, overestimating the amount of hours of wearing the AFO as we asked the patients to wear it as often as possible. Secondly, patients might have trouble to accurately recall the amount of wear hours, thereby inducing an overestimation of wear time. As the reliability of self-reported measures seems to be questionable, such data should be interpreted with care.

References:

[1] Lee S et al. Int J Rheum Dis, 2015.

[2] Duivenvoorden T et al. The Cochrane Library, 2015.

[3] Brouwer RW et al. Cochrane Database Syst Rev, 2005.

Disclosure of Interest: None declared

DOI: 10.1136/annrheumdis-2017-eular.1415

\section{AB0801 EVALUATION OF EFFECTIVITY OF RADIOTHERAPY FOR PAIN CONTROL IN OSTEOARTHRITIS AND SOFT TISSUE PATHOLOGY}

F. Aramburu, Á. Montero, R. Silvia, J. González, E. Calvo, M. Valero, C. Rubio, P. Garcia de la Peña on behalf of Rheumatology and Radiotherapy services. Rheumatology, HM hospitales, Madrid, Spain

Background: Osteoarthritis and soft tissue pathology are diseases that produce important pain and disability in many patients. Some studies support that the low-dose radiotherapy may be effective in refractory diseases.

Objectives: The main objective of this study is to evaluate whether the treatment with radiotherapy is long-term effective to reduce the pain in osteoarthritis and soft tissue pathology. The secondary objective is to evaluate the analgesic requirements before and after the treatment.

Methods: A prospective and observational study was designed. 38 patients were included. 53 different pathologies were treated including knee osteoarthritis, trochanteric bursitis, calcinosis, rizartrosis, pyramidal syndrome, rotatory cuff pathology and shoulder osteoarthritis, plantar fasciitis, epicondylitis, hand osteoarthritis, tibialis posterior tendonitis, ankle osteoarthritis, hip osteoarthritis and hand tendonitis. All these pathologies did not respond to conventional therapy (non steroidal antiinflamatory drugs, rehabilitation or infiltrations). Initially, the patients received a treatment of radiotherapy with a dose of 6 Gray divided in 6 sessions during 2 weeks. The patients that did not respond after 6 weeks received a second treatment with the same characteristics. The modification in Visual Analogic Scale (VAS) of pain was evaluated as well as analgesic intake before and after the treatment.

Results: No side effects were observed. $92 \%$ were women and $8 \%$ men. Mean age was 66,82, standard deviation (SD): 12,97 (42-89). 44 locations responded to one treatment and 19 needed a second one.

The mean VAS prior treatment was 7,38, SD: 1,49, (10-4). Mean VAS after the first treatment was 3,81 SD: $2,84(9-0)$.

The mean VAS after the first treatment in locations that received 2 treatments was 6,65 SD: 1,46 (9-5), and mean VAS after the second one was 4,56 DE: 2,66 (8-1).

By pathology, the difference in means was: 4,8 in knee osteoarthritis $(\mathrm{N}=11)$, 4,78 in trochanteric bursitis $(\mathrm{N}=10), 5$ in calcinosis $(\mathrm{N}=3), 5,33$ rizartrosis $(\mathrm{N}=8)$, 4 in pyramidal syndrome $(\mathrm{N}=2), 3,87$ in rotatory cuff pathology and shoulder osteoarthritis $(\mathrm{N}=6), 6$ in plantar fasciitis $(\mathrm{N}=1), 7$ in epicondylitis $(\mathrm{N}=1), 3,22$ in hand osteoarthritis $(\mathrm{N}=11), 6$ in tibialis posterior tendonitis $(\mathrm{N}=2), 4$ in ankle osteoarthritis (N=2), 2 in hip osteoarthritis $(\mathrm{N}=1)$ and 2,2 in hand tendonitis $(\mathrm{N}=5)$. Until November 2016, 12 patients (17 pathologies) were followed-up for 6 months. The mean VAS before treatment of these pathologies was 7,29 SD: 1,21; (9-4). After 6 months mean VAS reduced to 1,47 SD: 1,$42 ;(5-0)$.
The analgesic intake was reduced in $37,98 \%$ of patients, $10,34 \%$ used the same amount and $34,48 \%$ was not known. $17,24 \%$ did not take analgesic drugs at all after treatment.

Conclusions: The treatment with low dose radiotherapy was long-term effective for reducing the pain level in our study.

References:

[1] Minten MJ, Mahler E, den Broeder AA et al. The efficacy and safety of low-dose radiotherapy on pain and functioning in patients with osteoarthritis: a systematic review. Rheumatol Int. 2016 Jan; 36(1):133-42.

Disclosure of Interest: None declared

DOI: 10.1136/annrheumdis-2017-eular.4256

\section{AB0802 EFFECTS OF DIETARY MAGNESIUM SUPPLEMENTATION AND INTRA-ARTICULAR MAGNESIUM SULFATE ON EXPERIMENTAL OSTEOARTHRITIS AND POTENTIAL MOLECULAR MECHANISMS BY MRNA AND LNCRNA EXPRESSION PROFILES SCREENING}

G. Lei ${ }^{1}$, C. Zeng ${ }^{1}$, H. Li $^{1}$, J. Wei ${ }^{2}$, T. Yang ${ }^{1}$, B. Wise ${ }^{3}$, X. Ding ${ }^{1}$, Y. Zhang ${ }^{1}$ Y. Yang ${ }^{1}$, Z. Deng ${ }^{1}$, J. Li ${ }^{4}$, Y. Cui ${ }^{5} .{ }^{1}$ Department of Orthopaedics; ${ }^{2}$ Health Management Center, Xiangya Hospital of Central South University, Changsha, Hunan Province, China; ${ }^{3}$ Department of Internal Medicine and Department of Orthopaedics, University of California, Davis School of Medicine, Sacramento, $C A ;{ }^{4}$ Division of Rheumatology, Department of Internal Medicine, Rush University Medical Center, Chicago, IL, United States: ${ }^{5}$ International Medical Center, Xiangya Hospital, Central South University, Changsha, Hunan Province, China

Background: Epidemiological studies of ours and other groups have reported an inverse association between dietary and serum $\mathrm{Mg}$ with knee radiographic osteoarthritis $(\mathrm{OA})[1-4]$.

Objectives: To investigate the effects of dietary magnesium supplementation and intra-articular $\mathrm{MgSO}_{4}$ on the development of experimental rat osteoarthritis, and explore the underlying potential molecular mechanisms by mRNA and IncRNA expression profiles screening.

Methods: Rat osteoarthritis model was induced by surgery. Articular cartilage damage was evaluated by modified Mankin score system after intervention of dietary magnesium supplementation or intra-articular $\mathrm{MgSO}_{4}$ injection. Microarray was performed to reveal alteration of expression profiles of mRNA and IncRNA after intervention of $\mathrm{MgSO}_{4}$ on human osteoarthritis chondrocytes. Bioinformatics analyses including gene ontology analysis, pathway analysis, target gene predictions and network analysis were used.

Results: Comparing with normal diet, dietary $\mathrm{Mg}$ supplementation showed significantly ameliorated cartilage damage at the medial femoral condyle, lateral tibial plateau and medial tibial plateau $(P<0.05)$, and approaching significance at the lateral femoral condyle $(P=0.06)$. All four locations exhibited mitigated cartilage damage in the intra-articular $\mathrm{MgSO}_{4}$ group compared with intra-articular saline $(P \leq 0.05) .1767$ IncRNAs and 2558 mRNAs were upregulated while 994 IncRNAs and 1512 mRNAs were downregulated in chondrocytes with intervention of $50 \mathrm{mM} \mathrm{MgSO} 4$ compared with control group (fold change $\geq 2.0$ ). The top 6 IncRNAs which showed the largest difference were ENST00000425914.2, ENST00000419881.1, ENST00000561231.1, ENST00000609062.1, TCONS_00029212 and ENST00000429530.1. Bioinformatics analyses indicated that the differentially expressed IncRNA target genes of chondrocyte after intervention of $50 \mathrm{mM} \mathrm{MgSO}{ }_{4}$ are enriched in negative regulation of phosphatidylinositol 3-kinase signaling.

Conclusions: Both dietary magnesium supplementation and intra-articular $\mathrm{MgSO}_{4}$ injection may exert cartilage protective effects by causing widespread changes in the profile of IncRNAs and mRNAs of chondrocytes.

References:

[1] Hunter DJ. Evidence of altered bone turnover, vitamin $D$ and calcium regulation with knee osteoarthritis in female twins. Rheumatology 2003:42(11):1311-6.

[2] Zeng C, Wei J, Li H, Yang T, Zhang FJ, Pan D, et al. Relationship between Serum Magnesium Concentration and Radiographic Knee Osteoarthritis. J Rheumatol 2015;42(7):1231-6.

[3] Qin B, Shi X, Samai PS, Renner JB, Jordan JM, He K. Association of dietary magnesium intake with radiographic knee osteoarthritis: Results from a population-based study. Arthritis Care \& Research 2012;64(9):1306-11.

[4] Zeng C, Li H, Wei J, Yang T, Deng Z, Yang Y, et al. Association between Dietary Magnesium Intake and Radiographic Knee Osteoarthritis. PLOS ONE 2015;10(5):e127666.

Disclosure of Interest: None declared

DOI: 10.1136/annrheumdis-2017-eular.4647

\section{AB0803 DOES INTRA-ARTICULAR INJECTION OF PLATELET-RICH PLASMA PROVIDE CLINICALLY PREFERABLE OUTCOMES IN THE KNEE OSTEOARTHRITIS? A DOUBLE-BLIND, RANDOMIZED CONTROLLED PILOT STUDY}

J. Moghimi ${ }^{1,2}$, R. Ghorbani ${ }^{1}$, H. Beiki ${ }^{1}$. ${ }^{1}$ Social Determinants of Health Research Center; ${ }^{2}$ Department of Internal Medicine, Semnan university of medical sciences, Semnan, Iran, Islamic Republic Of

Background: Knee osteoarthritis (OA) symptoms Improvements with platelet- 